New Thiophene-Linked Conjugated Poly(azomethine)s: Theoretical

\title{
Electronic Structure, Synthesis, and Properties
}

Fu-Chuan Tsai, ${ }^{1}$ Chao-Ching Chang, ${ }^{1}$ Cheng-Liang Liu, ${ }^{1}$ Wen-Chang Chen, ${ }^{1 *}$ and Samson A. Jenekhe ${ }^{2 *}$

1 Department of Chemical Engineering and Institute of Polymer Science and Engineering, National Taiwan University, Taipei, Taiwan 106

2 Departments of Chemical Engineering and of Chemistry, University of Washington, Seattle, Washington 98195-1750

* For correspondence. E-mail: chenwc@ ntu.edu.tw ;

E-mail:jenekhe@u.washington.edu 
Table S1. The optimized geometry of PPI.

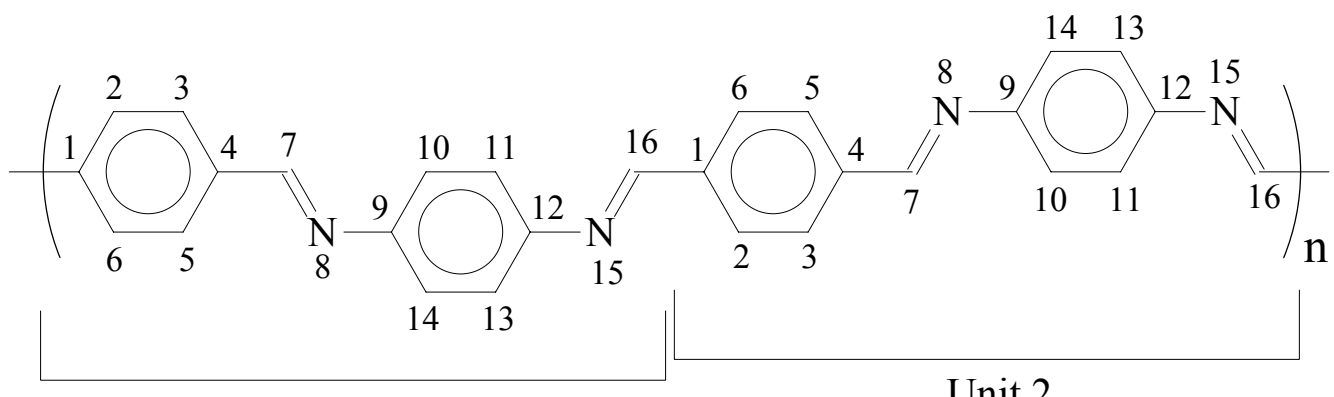

Unit 1

Unit 2

Bond length $(\AA)$

\begin{tabular}{llll}
\hline & Unit 1/ Unit 2 & & Unit 1/ Unit 2 \\
\hline $\mathrm{r}(1,2)$ & $1.410 / 1.410$ & $\mathrm{r}(9,10)$ & $1.411 / 1.411$ \\
$\mathrm{r}(2,3)$ & $1.390 / 1.390$ & $\mathrm{r}(10,11)$ & $1.393 / 1.393$ \\
$\mathrm{r}(3,4)$ & $1.411 / 1.411$ & $\mathrm{r}(11,12)$ & $1.411 / 1.411$ \\
$\mathrm{r}(4,5)$ & $1.410 / 1.410$ & $\mathrm{r}(12,13)$ & $1.409 / 1.409$ \\
$\mathrm{r}(5,6)$ & $1.390 / 1.390$ & $\mathrm{r}(13,14)$ & $1.390 / 1.390$ \\
$\mathrm{r}(6,1)$ & $1.412 / 1.411$ & $\mathrm{r}(14,9)$ & $1.409 / 1.409$ \\
$\mathrm{r}(4,7)$ & $1.463 / 1.463$ & $\mathrm{r}(12,15)$ & $1.411 / 1.410$ \\
$\mathrm{r}(7,8)$ & $1.293 / 1.293$ & $\mathrm{r}(15,16)$ & $1.293 / 1.293$ \\
$\mathrm{r}(8,9)$ & $1.410 / 1.410$ & $\mathrm{r}(16,1)$ & $1.463 /-$ \\
\hline
\end{tabular}

Bond angle (degree)

\begin{tabular}{ll|ll}
\hline & Unit 1 / Unit 2 & & Unit 1 / Unit 2 \\
\hline$\theta(3,4,7)$ & $119.6 / 119.6$ & $\theta(11,12,15)$ & $129.3 / 129.3$ \\
$\theta(5,4,7)$ & $121.4 / 121.4$ & $\theta(13,12,15)$ & $117.3 / 117.3$ \\
$\theta(4,7,8)$ & $122.4 / 122.4$ & $\theta(12,15,16)$ & $122.5 / 122.5$ \\
$\theta(7,8,9)$ & $122.5 / 122.5$ & $\theta(15,16,1)$ & $122.4 /-$ \\
$\theta(8,9,10)$ & $123.9 / 123.9$ & $\theta(16,1,2)$ & $121.4 /-$ \\
$\theta(8,9,14)$ & $117.3 / 117.3$ & $\theta(16,1,6)$ & $119.6 /-$ \\
\hline
\end{tabular}

Dihedral angle (degree)

\begin{tabular}{ll|ll}
\hline & Unit 1 / Unit 2 & & Unit 1 / Unit 2 \\
\hline$\Phi(3,4,7,8)$ & $0.9 / 0.8$ & $\Phi(11,12,15,16)$ & $31.8 / 31.9$ \\
$\Phi(5,4,7,8)$ & $1.0 / 0.9$ & $\Phi(13,12,15,16)$ & $29.0 / 29.0$ \\
$\Phi(7,8,9,10)$ & $31.9 / 31.8$ & $\Phi(15,16,1,2)$ & $0.9 /-$ \\
$\Phi(7,8,9,14)$ & $29.0 / 28.9$ & $\Phi(15,16,1,6)$ & $0.8 /-$ \\
\hline
\end{tabular}


Table S2. The optimized geometry of PHTPI treated without 3-hexyl side-group on the thiophene ring.

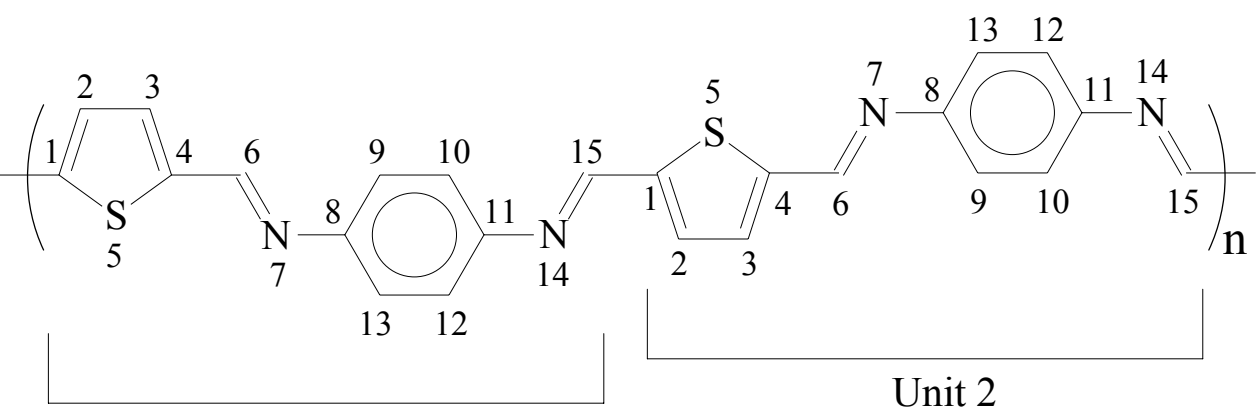

Unit 1

Bond length $(\AA)$

\begin{tabular}{llll}
\hline & Unit 1 / Unit 2 & & Unit 1 / Unit 2 \\
\hline $\mathrm{r}(1,2)$ & $1.382 / 1.382$ & $\mathrm{r}(9,10)$ & $1.391 / 1.391$ \\
$\mathrm{r}(2,3)$ & $1.416 / 1.416$ & $\mathrm{r}(10,11)$ & $1.413 / 1.414$ \\
$\mathrm{r}(3,4)$ & $1.385 / 1.385$ & $\mathrm{r}(11,12)$ & $1.410 / 1.410$ \\
$\mathrm{r}(4,5)$ & $1.809 / 1.809$ & $\mathrm{r}(12,13)$ & $1.389 / 1.389$ \\
$\mathrm{r}(1,5)$ & $1.816 / 1.816$ & $\mathrm{r}(8,13)$ & $1.410 / 1.410$ \\
$\mathrm{r}(4,6)$ & $1.435 / 1.435$ & $\mathrm{r}(11,14)$ & $1.406 / 1.406$ \\
$\mathrm{r}(6,7)$ & $1.297 / 1.297$ & $\mathrm{r}(14,15)$ & $1.298 / 1.298$ \\
$\mathrm{r}(7,8)$ & $1.407 / 1.407$ & $\mathrm{r}(15,1)$ & $1.443 /-$ \\
$\mathrm{r}(8,9)$ & $1.413 / 1.413$ & & \\
\hline
\end{tabular}

Bond angle (degree)

\begin{tabular}{ll|ll}
\hline & Unit 1 / Unit 2 & & Unit 1/ Unit 2 \\
\hline$\theta(3,4,6)$ & $127.6 / 127.6$ & $\theta(10,11,14)$ & $124.3 / 124.3$ \\
$\theta(5,4,6)$ & $121.3 / 121.3$ & $\theta(12,11,14)$ & $117.0 / 117.0$ \\
$\theta(4,6,7)$ & $122.4 / 122.4$ & $\theta(11,14,15)$ & $123.3 / 123.2$ \\
$\theta(6,7,8)$ & $122.7 / 122.7$ & $\theta(14,15,1)$ & $120.7 /-$ \\
$\theta(7,8,9)$ & $124.5 / 124.5$ & $\theta(15,1,2)$ & $128.4 /-$ \\
$\theta(7,8,13)$ & $116.8 / 116.8$ & $\theta(15,1,5)$ & $120.3 /-$ \\
\hline
\end{tabular}

Dihedral angle (degree)

\begin{tabular}{ll|ll}
\hline & Unit 1 / Unit 2 & & Unit 1 / Unit 2 \\
\hline$\Phi(3,4,6,7)$ & $1.6 / 1.5$ & $\Phi(10,11,14,15)$ & $25.0 / 25.3$ \\
$\Phi(5,4,6,7)$ & $0.4 / 0.5$ & $\Phi(12,11,14,15)$ & $22.5 / 22.8$ \\
$\Phi(6,7,8,9)$ & $25.2 / 24.9$ & $\Phi(14,15,1,2)$ & $01 /-$ \\
$\Phi(6,7,8,13)$ & $25.4 / 25.0$ & $\Phi(14,15,1,5)$ & $2.2 /-$ \\
\hline
\end{tabular}


Table S3. The optimized geometry of PHTFI treated without 3-hexyl side-group on the thiophene ring.

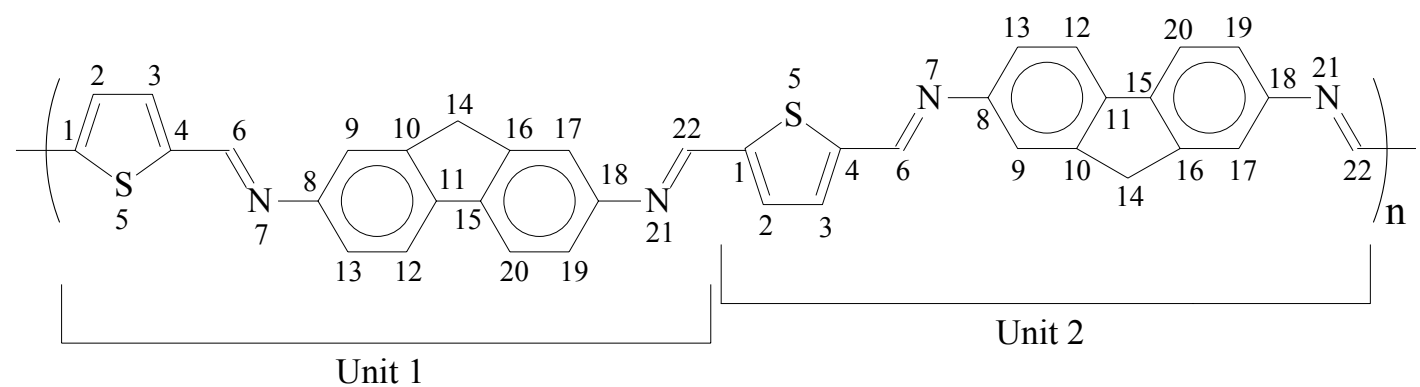

Bond length $(\AA)$

\begin{tabular}{ll|ll}
\hline & Unit 1/ Unit 2 & & Unit 1/ Unit 2 \\
\hline $\mathrm{r}(1,2)$ & $1.382 / 1.382$ & $\mathrm{r}(8,13)$ & $1.412 / 1.412$ \\
$\mathrm{r}(2,3)$ & $1.417 / 1.417$ & $\mathrm{r}(10,14)$ & $1.521 / 1.521$ \\
$\mathrm{r}(3,4)$ & $1.384 / 1.384$ & $\mathrm{r}(11,15)$ & $1.465 / 1.465$ \\
$\mathrm{r}(4,5)$ & $1.809 / 1.809$ & $\mathrm{r}(14,16)$ & $1.521 / 1.521$ \\
$\mathrm{r}(1,5)$ & $1.816 / 1.816$ & $\mathrm{r}(15,16)$ & $1.419 / 1.419$ \\
$\mathrm{r}(4,6)$ & $1.436 / 1.436$ & $\mathrm{r}(16,17)$ & $1.387 / 1.387$ \\
$\mathrm{r}(6,7)$ & $1.297 / 1.297$ & $\mathrm{r}(17,18)$ & $1.416 / 1.416$ \\
$\mathrm{r}(7,8)$ & $1.408 / 1.408$ & $\mathrm{r}(18,19)$ & $1.412 / 1.412$ \\
$\mathrm{r}(8,9)$ & $1.415 / 1.415$ & $\mathrm{r}(19,20)$ & $1.394 / 1.395$ \\
$\mathrm{r}(9,10)$ & $1.388 / 1.388$ & $\mathrm{r}(15,20)$ & $1.401 / 1.401$ \\
$\mathrm{r}(10,11)$ & $1.419 / 1.419$ & $\mathrm{r}(18,21)$ & $1.409 / 1.409$ \\
$\mathrm{r}(11,12)$ & $1.401 / 1.401$ & $\mathrm{r}(21,22)$ & $1.298 / 1.298$ \\
$\mathrm{r}(12,13)$ & $1.394 / 1.394$ & $\mathrm{r}(22,1)$ & $1.443 /-$ \\
\hline
\end{tabular}

Bond angle (degree)

\begin{tabular}{llll}
\hline & Unit 1 / Unit 2 & & Unit 1 / Unit 2 \\
\hline$\theta(3,4,6)$ & $127.9 / 127.9$ & $\theta(17,18,21)$ & $124.0 / 124.0$ \\
$\theta(5,4,6)$ & $121.0 / 121.0$ & $\theta(19,18,21)$ & $116.4 / 116.4$ \\
$\theta(4,6,7)$ & $122.2 / 122.2$ & $\theta(18,21,22)$ & $122.7 / 122.7$ \\
$\theta(6,7,8)$ & $122.9 / 122.9$ & $\theta(21,22,1)$ & $121.0 /-$ \\
$\theta(7,8,9)$ & $123.8 / 123.9$ & $\theta(22,1,2)$ & $128.7 /-$ \\
$\theta(7,8,13)$ & $116.5 / 116.5$ & $\theta(22,1,5)$ & $120.0 /-$ \\
\hline
\end{tabular}

Dihedral angle (degree)

\begin{tabular}{ll|ll}
\hline & Unit 1 / Unit 2 & & Unit 1 / Unit 2 \\
\hline$\Phi(3,4,6,7)$ & $1.3 / 1.3$ & $\Phi(17,18,21,22)$ & $25.8 / 25.9$ \\
$\Phi(5,4,6,7)$ & $1.1 / 1.1$ & $\Phi(19,18,21,22)$ & $24.0 / 24.0$ \\
$\Phi(6,7,8,9)$ & $28.2 . / 28.0$ & $\Phi(21,22,1,2)$ & $0.6 /-$ \\
$\Phi(6,7,8,13)$ & $26.3 / 26.1$ & $\Phi(21,22,1,5)$ & $0.3 /-$ \\
\hline
\end{tabular}


Table S4. The optimized geometry of PHTCI treated without 3-hexyl side-group on the thiophene ring.

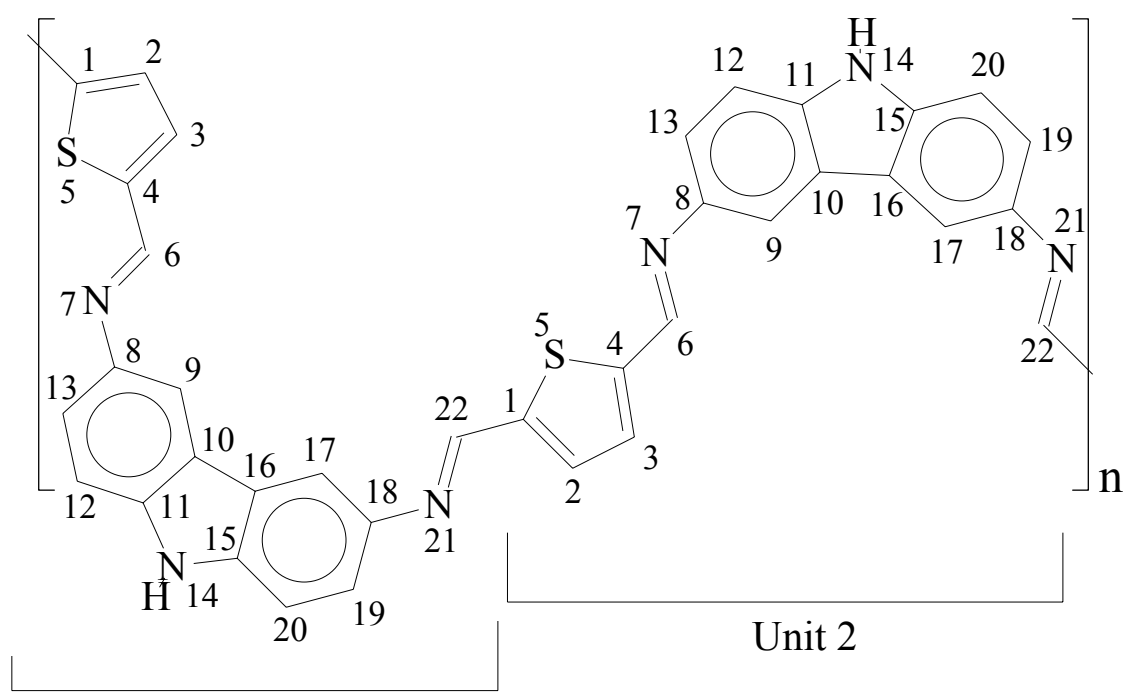

Unit 1

Bond length $(\AA)$

\begin{tabular}{ll|ll}
\hline & Unit 1/ Unit 2 & & Unit 1/ Unit 2 \\
\hline $\mathrm{r}(1,2)$ & $1.381 / 1.381$ & $\mathrm{r}(8,13)$ & $1.418 / 1.418$ \\
$\mathrm{r}(2,3)$ & $1.418 / 1.418$ & $\mathrm{r}(11,14)$ & $1.395 / 1.395$ \\
$\mathrm{r}(3,4)$ & $1.384 / 1.384$ & $\mathrm{r}(14,15)$ & $1.394 / 1.394$ \\
$\mathrm{r}(4,5)$ & $1.810 / 1.810$ & $\mathrm{r}(10,16)$ & $1.457 / 1.457$ \\
$\mathrm{r}(1,5)$ & $1.817 / 1.817$ & $\mathrm{r}(15,16)$ & $1.427 / 1.427$ \\
$\mathrm{r}(4,6)$ & $1.436 / 1.436$ & $\mathrm{r}(16,17)$ & $1.396 / 1.396$ \\
$\mathrm{r}(6,7)$ & $1.296 / 1.296$ & $\mathrm{r}(17,18)$ & $1.408 / 1.408$ \\
$\mathrm{r}(7,8)$ & $1.411 / 1.411$ & $\mathrm{r}(18,19)$ & $1.418 / 1.418$ \\
$\mathrm{r}(8,9)$ & $1.408 / 1.408$ & $\mathrm{r}(19,20)$ & $1.391 / 1.391$ \\
$\mathrm{r}(9,10)$ & $1.397 / 1.397$ & $\mathrm{r}(15,20)$ & $1.399 / 1.399$ \\
$\mathrm{r}(10,11)$ & $1.426 / 1.426$ & $\mathrm{r}(18,21)$ & $1.411 / 1.411$ \\
$\mathrm{r}(11,12)$ & $1.399 / 1.399$ & $\mathrm{r}(21,22)$ & $1.297 / 1.297$ \\
$\mathrm{r}(12,13)$ & $1.392 / 1.392$ & $\mathrm{r}(22,1)$ & $1.443 /-$ \\
\hline
\end{tabular}

Bond angle (degree)

\begin{tabular}{ll|ll}
\hline & Unit 1 / Unit 2 & & Unit 1 / Unit 2 \\
\hline$\theta(3,4,6)$ & $127.8 / 127.9$ & $\theta(17,18,21)$ & $124.5 / 124.7$ \\
$\theta(5,4,6)$ & $121.2 / 121.2$ & $\theta(19,18,21)$ & $115.8 / 115.7$ \\
$\theta(4,6,7)$ & $122.3 / 122.3$ & $\theta(18,21,22)$ & $123.1 / 123.2$ \\
$\theta(6,7,8)$ & $122.7 / 122.6$ & $\theta(21,22,1)$ & $120.9 /-$ \\
$\theta(7,8,9)$ & $124.1 / 124.0$ & $\theta(22,1,2)$ & $128.8 /-$ \\
$\theta(7,8,13)$ & $116.1 / 116.2$ & $\theta(22,1,5)$ & $120.0 /-$ \\
\hline
\end{tabular}

Dihedral angle (degree)

\begin{tabular}{ll|ll}
\hline & Unit 1 / Unit 2 & & Unit 1 / Unit 2 \\
\hline$\Phi(3,4,6,7)$ & $2.2 / 2.1$ & $\Phi(17,18,21,22)$ & $21.6 / 20.5$ \\
$\Phi(5,4,6,7)$ & $1.6 / 1.6$ & $\Phi(19,18,21,22)$ & $29.8 / 28.7$ \\
$\Phi(6,7,8,9)$ & $27.8 / 28.2$ & $\Phi(21,22,1,2)$ & $0.3 /-$ \\
$\Phi(6,7,8,13)$ & $26.2 / 26.6$ & $\Phi(21,22,1,5)$ & $0.6 /-$ \\
\hline \multicolumn{5}{l}{5}
\end{tabular}


Table S5. The optimized geometry of PHTNI treated without 3-hexyl side-group on the thiophene ring.

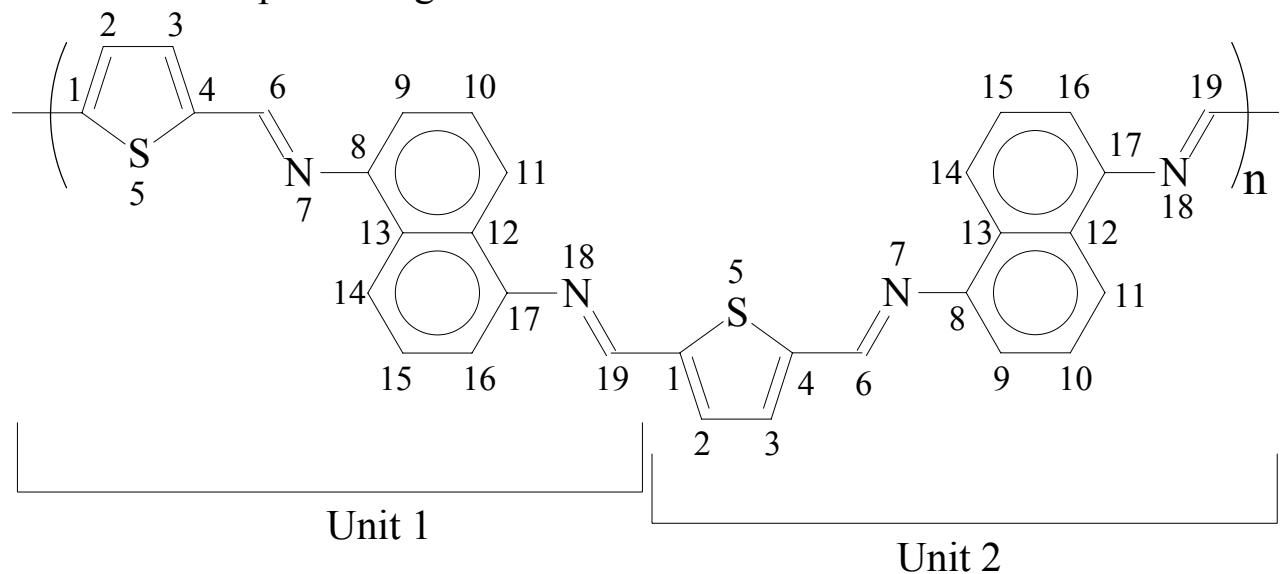

Bond length $(\AA)$

\begin{tabular}{ll|ll}
\hline & Unit 1 / Unit 2 & & Unit 1/ Unit 2 \\
\hline $\mathrm{r}(1,2)$ & $1.383 / 1.383$ & $\mathrm{r}(11,12)$ & $1.421 / 1.421$ \\
$\mathrm{r}(2,3)$ & $1.419 / 1.419$ & $\mathrm{r}(12,13)$ & $1.434 / 1.434$ \\
$\mathrm{r}(3,4)$ & $1.383 / 1.383$ & $\mathrm{r}(8,13)$ & $1.439 / 1.439$ \\
$\mathrm{r}(4,5)$ & $1.812 / 1.812$ & $\mathrm{r}(13,14)$ & $1.421 / 1.421$ \\
$\mathrm{r}(1,5)$ & $1.812 / 1.812$ & $\mathrm{r}(14,15)$ & $1.382 / 1.382$ \\
$\mathrm{r}(4,6)$ & $1.437 / 1.437$ & $\mathrm{r}(15,16)$ & $1.411 / 1.411$ \\
$\mathrm{r}(6,7)$ & $1.296 / 1.296$ & $\mathrm{r}(16,17)$ & $1.393 / 1.393$ \\
$\mathrm{r}(7,8)$ & $1.407 / 1.407$ & $\mathrm{r}(17,12)$ & $1.440 / 1.439$ \\
$\mathrm{r}(8,9)$ & $1.393 / 1.393$ & $\mathrm{r}(17,18)$ & $1.407 / 1.407$ \\
$\mathrm{r}(9,10)$ & $1.411 / 1.411$ & $\mathrm{r}(18,19)$ & $1.296 / 1.296$ \\
$\mathrm{r}(10,11)$ & $1.383 / 1.382$ & $\mathrm{r}(19,1)$ & $1.437 /-$ \\
\hline
\end{tabular}

Bond angle (degree)

\begin{tabular}{ll|ll}
\hline & Unit 1 / Unit 2 & & Unit 1 / Unit 2 \\
\hline$\theta(3,4,6)$ & $127.2 / 127.2$ & $\theta(12,17,18)$ & $117.0 / 117.1$ \\
$\theta(5,4,6)$ & $121.3 / 121.3$ & $\theta(16,17,18)$ & $123.2 / 123.1$ \\
$\theta(4,6,7)$ & $122.3 / 122.3$ & $\theta(17,18,19)$ & $122.7 / 122.6$ \\
$\theta(6,7,8)$ & $122.7 / 122.6$ & $\theta(18,19,1)$ & $122.4 /-$ \\
$\theta(7,8,9)$ & $123.2 / 123.1$ & $\theta(19,1,2)$ & $127.2 /-$ \\
$\theta(7,8,13)$ & $117.0 / 117.1$ & $\theta(19,1,5)$ & $121.3 /-$ \\
\hline
\end{tabular}

Dihedral angle (degree)

\begin{tabular}{ll|ll}
\hline & Unit 1 / Unit 2 & & Unit 1 / Unit 2 \\
\hline$\Phi(3,4,6,7)$ & $0.8 / 0.8$ & $\Phi(12,17,18,19)$ & $29.2 / 29.4$ \\
$\Phi(5,4,6,7)$ & $0.6 / 0.8$ & $\Phi(16,17,18,19)$ & $31.7 / 32.0$ \\
$\Phi(6,7,8,9)$ & $31.6 / 31.9$ & $\Phi(18,19,1,2)$ & $0.8 /-$ \\
$\Phi(6,7,8,13)$ & $29.1 / 29.3$ & $\Phi(18,19,1,5)$ & $0.7 /-$ \\
\hline
\end{tabular}


Table S6. The optimized geometry of PHTMOBPI treated without 3-hexyl side-group on the thiophene ring.

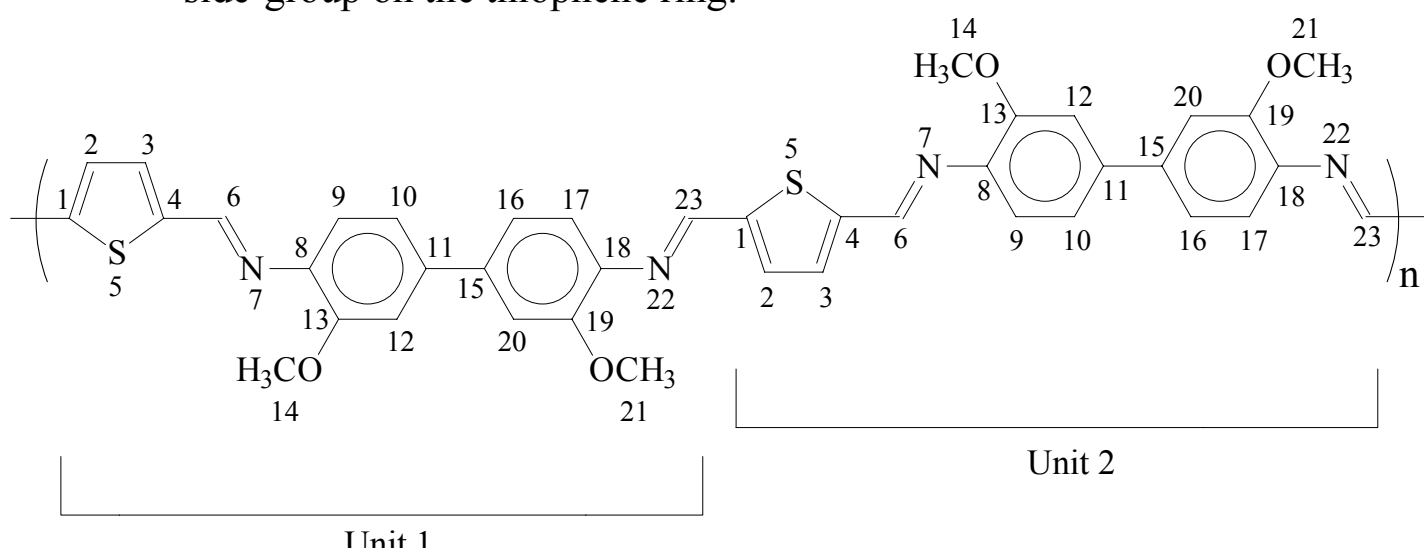

Bond length $(\AA)$

\begin{tabular}{ll|ll}
\hline & Unit 1/ Unit 2 & & Unit 1/ Unit 2 \\
\hline $\mathrm{r}(1,2)$ & $1.382 / 1.382$ & $\mathrm{r}(8,13)$ & $1.421 / 1.421$ \\
$\mathrm{r}(2,3)$ & $1.417 / 1.417$ & $\mathrm{r}(13,14)$ & $1.380 / 1.380$ \\
$\mathrm{r}(3,4)$ & $1.385 / 1.385$ & $\mathrm{r}(11,15)$ & $1.482 / 1.482$ \\
$\mathrm{r}(4,5)$ & $1.811 / 1.811$ & $\mathrm{r}(15,16)$ & $1.412 / 1.413$ \\
$\mathrm{r}(1,5)$ & $1.818 / 1.818$ & $\mathrm{r}(16,17)$ & $1.389 / 1.389$ \\
$\mathrm{r}(4,6)$ & $1.435 / 1.435$ & $\mathrm{r}(17,18)$ & $1.413 / 1.413$ \\
$\mathrm{r}(6,7)$ & $1.298 / 1.298$ & $\mathrm{r}(18,19)$ & $1.419 / 1.419$ \\
$\mathrm{r}(7,8)$ & $1.403 / 1.403$ & $\mathrm{r}(19,20)$ & $1.397 / 1.397$ \\
$\mathrm{r}(8,9)$ & $1.416 / 1.417$ & $\mathrm{r}(15,20)$ & $1.404 / 1.404$ \\
$\mathrm{r}(9,10)$ & $1.386 / 1.386$ & $\mathrm{r}(19,21)$ & $1.390 / 1.390$ \\
$\mathrm{r}(10,11)$ & $1.415 / 1.415$ & $\mathrm{r}(18,22)$ & $1.406 / 1.406$ \\
$\mathrm{r}(11,12)$ & $1.399 / 1.399$ & $\mathrm{r}(22,23)$ & $1.299 / 1.299$ \\
$\mathrm{r}(12,13)$ & $1.404 / 1.404$ & $\mathrm{r}(23,1)$ & $1.442 /-$ \\
\hline
\end{tabular}

Bond angle (degree)

\begin{tabular}{ll|ll}
\hline & Unit 1 / Unit 2 & & Unit 1/ Unit 2 \\
\hline$\theta(3,4,6)$ & $127.8 / 127.8$ & $\theta(11,15,16)$ & $120.9 / 120.9$ \\
$\theta(5,4,6)$ & $121.3 / 121.3$ & $\theta(11,15,20)$ & $121.1 / 121.1$ \\
$\theta(4,6,7)$ & $122.2 / 122.2$ & $\theta(18,19,21)$ & $123.7 / 123.7$ \\
$\theta(6,7,8)$ & $123.5 / 123.5$ & $\theta(17,18,22)$ & $124.4 / 124.5$ \\
$\theta(7,8,9)$ & $123.3 / 123.3$ & $\theta(19,18,22)$ & $118.0 / 117.9$ \\
$\theta(7,8,13)$ & $119.3 / 119.3$ & $\theta(18,22,23)$ & $123.5 / 123.6$ \\
$\theta(8,13,14)$ & $127.1 / 127.0$ & $\theta(22,23,1)$ & $120.9 /-$ \\
$\theta(10,11,15)$ & $120.9 / 120.9$ & $\theta(23,1,2)$ & $129.1 /-$ \\
$\theta(12,11,15)$ & $121.0 / 121.1$ & $\theta(23,1,5)$ & $119.9 /-$ \\
\hline
\end{tabular}

Dihedral angle (degree)

\begin{tabular}{ll|ll}
\hline & Unit 1 / Unit 2 & & Unit 1 / Unit 2 \\
\hline$\Phi(3,4,6,7)$ & $1.6 / 1.6$ & $\Phi(9,8,13,14)$ & $0.8 / 0.8$ \\
$\Phi(5,4,6,7)$ & $1.2 / 1.1$ & $\Phi(17,18,19,21)$ & $2.6 / 2.6$ \\
$\Phi(6,7,8,9)$ & $19.4 / 19.5$ & $\Phi(17,18,22,23)$ & $16.0 / 14.4$ \\
$\Phi(6,7,8,13)$ & $18.2 / 18.2$ & $\Phi(19,18,22,23)$ & $12.7 / 11.3$ \\
$\Phi(10,11,15,16)$ & $36.4 / 36.5$ & $\Phi(22,23,1,2)$ & $0.9 /-$ \\
$\Phi(12,11,15,20)$ & $35.3 / 35.4$ & $\Phi(22,23,1,5)$ & $0.8 /-$ \\
\hline
\end{tabular}


Table S7. The optimized geometry of PHTMOPI treated without 3-hexyl side-group on the thiophene ring.

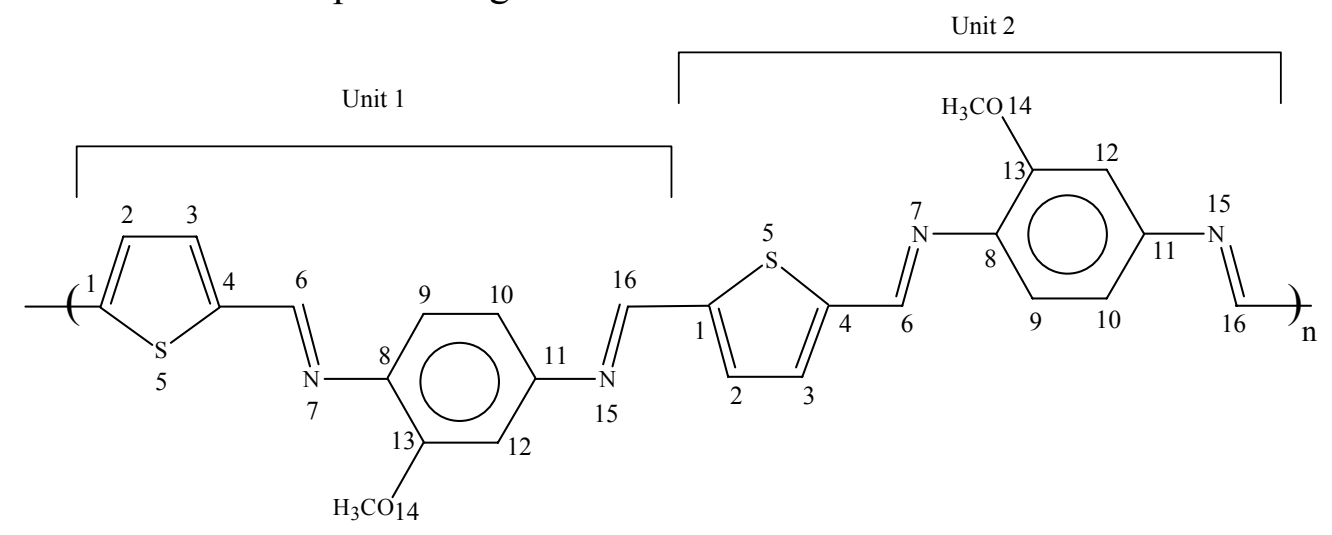

Bond length $(\AA)$

\begin{tabular}{lc|lr}
\hline & Unit 1 / Unit 2 & & Unit 1/ Unit 2 \\
\hline $\mathrm{r}(1,2)$ & $1.381 / 1.381$ & $\mathrm{r}(9,10)$ & $1.386 / 1.386$ \\
$\mathrm{r}(2,3)$ & $1.417 / 1.417$ & $\mathrm{r}(10,11)$ & $1.415 / 1.415$ \\
$\mathrm{r}(3,4)$ & $1.385 / 1.385$ & $\mathrm{r}(11,12)$ & $1.400 / 1.400$ \\
$\mathrm{r}(4,5)$ & $1.811 / 1.811$ & $\mathrm{r}(12,13)$ & $1.399 / 1.399$ \\
$\mathrm{r}(1,5)$ & $1.817 / 1.817$ & $\mathrm{r}(13,14)$ & $1.381 / 1.381$ \\
$\mathrm{r}(4,6)$ & $1.435 / 1.435$ & $\mathrm{r}(8,13)$ & $1.423 / 1.423$ \\
$\mathrm{r}(6,7)$ & $1.299 / 1.298$ & $\mathrm{r}(11,15)$ & $1.409 / 1.409$ \\
$\mathrm{r}(7,8)$ & $1.402 / 1.402$ & $\mathrm{r}(15,16)$ & $1.297 / 1.297$ \\
$\mathrm{r}(8,9)$ & $1.417 / 1.417$ & $\mathrm{r}(16,1)$ & $1.443 /-----$ \\
\hline
\end{tabular}

Bond angle (degree)

\begin{tabular}{lcll}
\hline & Unit 1 / Unit 2 & & Unit 1/ Unit 2 \\
\hline$\theta(3,4,6)$ & $127.8 / 127.8$ & $\theta(10,11,15)$ & $124.1 / 124.1$ \\
$\theta(5,4,6)$ & $121.2 / 121.2$ & $\theta(12,11,15)$ & $116.9 / 116.9$ \\
$\theta(4,6,7)$ & $122.3 / 122.3$ & $\theta(11,15,16)$ & $122.2 / 122.2$ \\
$\theta(6,7,8)$ & $122.8 / 122.8$ & $\theta(15,16,1)$ & $120.9 /-----$ \\
$\theta(7,8,9)$ & $122.7 / 122.7$ & $\theta(16,1,2)$ & $128.8 /-----$ \\
$\theta(7,8,13)$ & $119.5 / 119.5$ & $\theta(16,1,5)$ & $120.1 /----$ \\
$\theta(8,13,14)$ & $126.4 / 126.4$ & & \\
\hline
\end{tabular}

Dihedral angle (degree)

\begin{tabular}{lc|lc}
\hline & Unit 1 / Unit 2 & & Unit 1 / Unit 2 \\
\hline$\Phi(3,4,6,7)$ & $2.1 / 1.9$ & $\Phi(10,11,15,16)$ & $28.9 / 28.6$ \\
$\Phi(5,4,6,7)$ & $1.6 / 1.5$ & $\Phi(12,11,15,16)$ & $26.2 / 26.0$ \\
$\Phi(6,7,8,9)$ & $27.7 / 27.6$ & $\Phi(15,16,1,2)$ & $0.9 /----$ \\
$\Phi(6,7,8,13)$ & $25.8 / 25.7$ & $\Phi(15,16,1,5)$ & $1.6 /---$ \\
$\Phi(9,8,13,14)$ & $1.0 / 0.0$ & & \\
\hline
\end{tabular}


Table S8. The optimized geometry of PHTNOPI treated without 3-hexyl side-group on the thiophene ring.

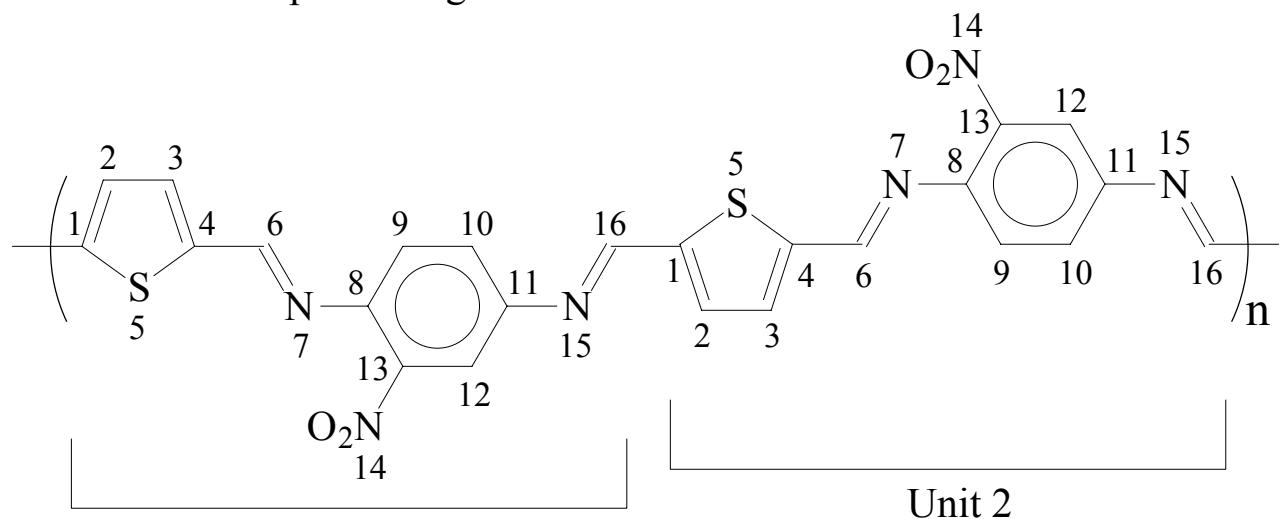

Unit 1

Bond length $(\AA)$

\begin{tabular}{ll|ll}
\hline & Unit 1/ Unit 2 & & Unit 1/ Unit 2 \\
\hline $\mathrm{r}(1,2)$ & $1.382 / 1.383$ & $\mathrm{r}(9,10)$ & $1.391 / 1.391$ \\
$\mathrm{r}(2,3)$ & $1.417 / 1.416$ & $\mathrm{r}(10,11)$ & $1.412 / 1.412$ \\
$\mathrm{r}(3,4)$ & $1.384 / 1.384$ & $\mathrm{r}(11,12)$ & $1.403 / 1.403$ \\
$\mathrm{r}(4,5)$ & $1.806 / 1.806$ & $\mathrm{r}(12,13)$ & $1.391 / 1.390$ \\
$\mathrm{r}(1,5)$ & $1.812 / 1.812$ & $\mathrm{r}(13,14)$ & $1.460 / 1.460$ \\
$\mathrm{r}(4,6)$ & $1.436 / 1.436$ & $\mathrm{r}(8,13)$ & $1.417 / 1.417$ \\
$\mathrm{r}(6,7)$ & $1.293 / 1.293$ & $\mathrm{r}(11,15)$ & $1.407 / 1.407$ \\
$\mathrm{r}(7,8)$ & $1.394 / 1.394$ & $\mathrm{r}(15,16)$ & $1.297 / 1.293$ \\
$\mathrm{r}(8,9)$ & $1.413 / 1.413$ & $\mathrm{r}(16,1)$ & $1.443 /-$ \\
\hline
\end{tabular}

Bond angle (degree)

\begin{tabular}{ll|ll}
\hline & Unit 1 / Unit 2 & & Unit 1/ Unit 2 \\
\hline$\theta(3,4,6)$ & $127.7 / 127.7$ & $\theta(10,11,15)$ & $124.1 / 124.1$ \\
$\theta(5,4,6)$ & $121.0 / 121.0$ & $\theta(12,11,15)$ & $117.3 / 117.3$ \\
$\theta(4,6,7)$ & $122.2 / 122.1$ & $\theta(11,15,16)$ & $122.1 / 122.1$ \\
$\theta(6,7,8)$ & $123.5 / 123.5$ & $\theta(15,16,1)$ & $121.1 /-$ \\
$\theta(7,8,9)$ & $121.7 / 121.7$ & $\theta(16,1,2)$ & $128.5 /-$ \\
$\theta(7,8,13)$ & $121.1 / 121.1$ & $\theta(16,1,5)$ & $119.9 /-$ \\
$\theta(8,13,14)$ & $120.7 / 120.7$ & & \\
\hline
\end{tabular}

Dihedral angle (degree)

\begin{tabular}{ll|ll}
\hline & Unit 1 / Unit 2 & & Unit 1 / Unit 2 \\
\hline$\Phi(3,4,6,7)$ & $2.5 / 2.6$ & $\Phi(10,11,15,16)$ & $33.6 / 33.6$ \\
$\Phi(5,4,6,7)$ & $2.6 / 2.6$ & $\Phi(12,11,15,16)$ & $31.1 / 31.1$ \\
$\Phi(6,7,8,9)$ & $45.9 / 46.1$ & $\Phi(15,16,1,2)$ & $4.9 /-$ \\
$\Phi(6,7,8,13)$ & $38.3 / 38.5$ & $\Phi(15,16,1,5)$ & $4.2 /-$ \\
$\Phi(9,8,13,14)$ & $1.5 / 1.5$ & & \\
\hline
\end{tabular}


Tabble S9. Assignment of the FTIR spectra of the poly(azomethine)s.

\begin{tabular}{lcccc}
\hline & $\mathrm{C}=\mathrm{N}$ stretch & $\mathrm{C}=\mathrm{O}$ stretch & aliphatic C-H stretch & aromatic C-H bending \\
\hline DFHT & - & 1678 & $2956,2929,2858$ & 867 \\
PHTPI & 1594 & - & $2954,2928,2857$ & 846 \\
PHTFI & 1591 & - & $2952,2927,2857$ & 855,823 \\
PHTCI & 1611 & - & $2953,2927,2857$ & 867,810 \\
PHTNI & 1597 & $1658^{\mathrm{a}}$ & $2953,2926,2855$ & 785 \\
PHTMOBPI & 1609 & $1670^{\mathrm{a}}$ & $2950,2927,2857$ & $795-848$ \\
\hline
\end{tabular}

a: residual aldehyde 


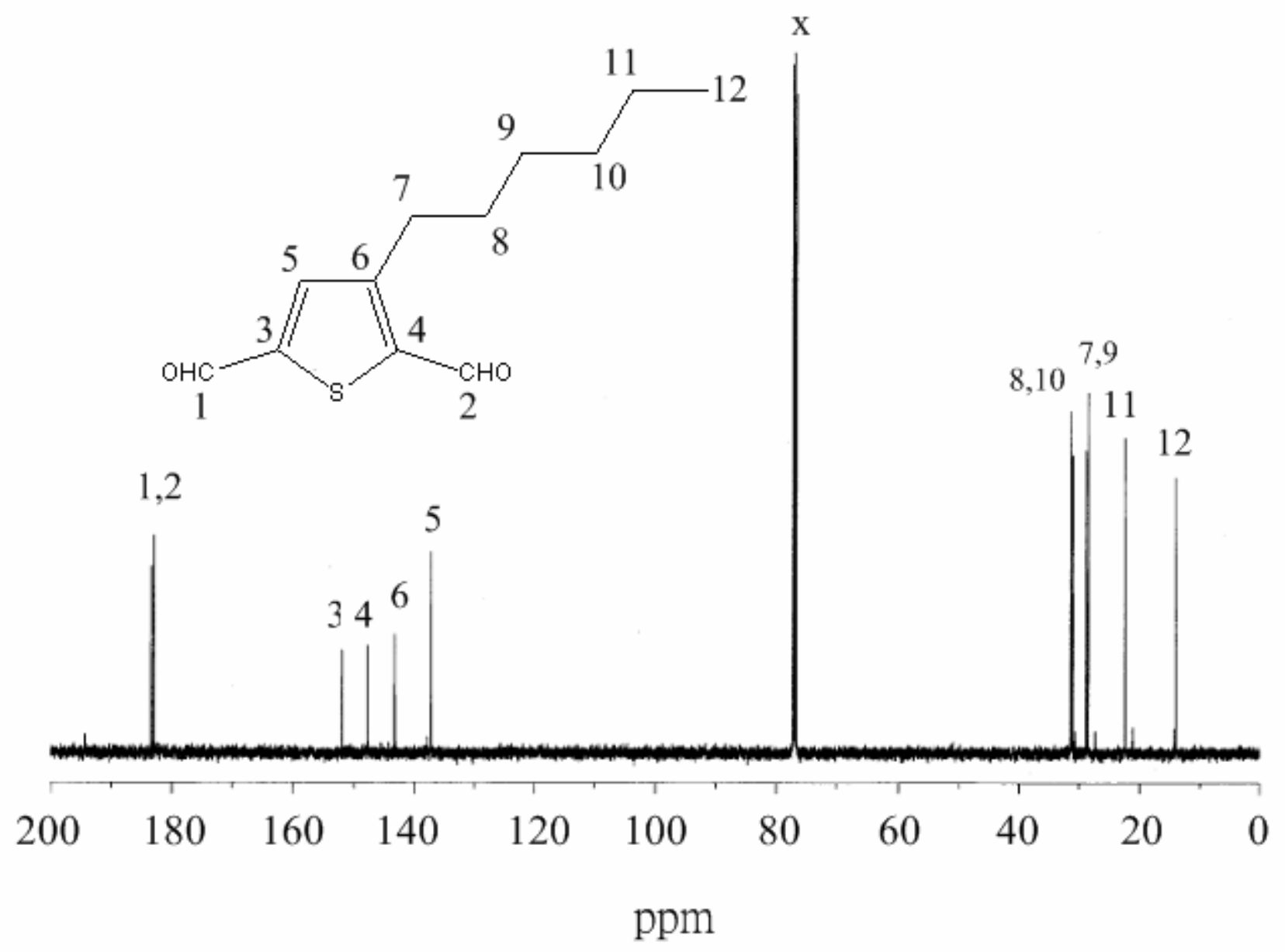

Figure S1. The ${ }^{13} \mathrm{C}$ NMR spectrum of monomer DFHT (in $\mathrm{CDCl}_{3}$ ). $\mathrm{x}: \mathrm{CHCl}_{3}$. 


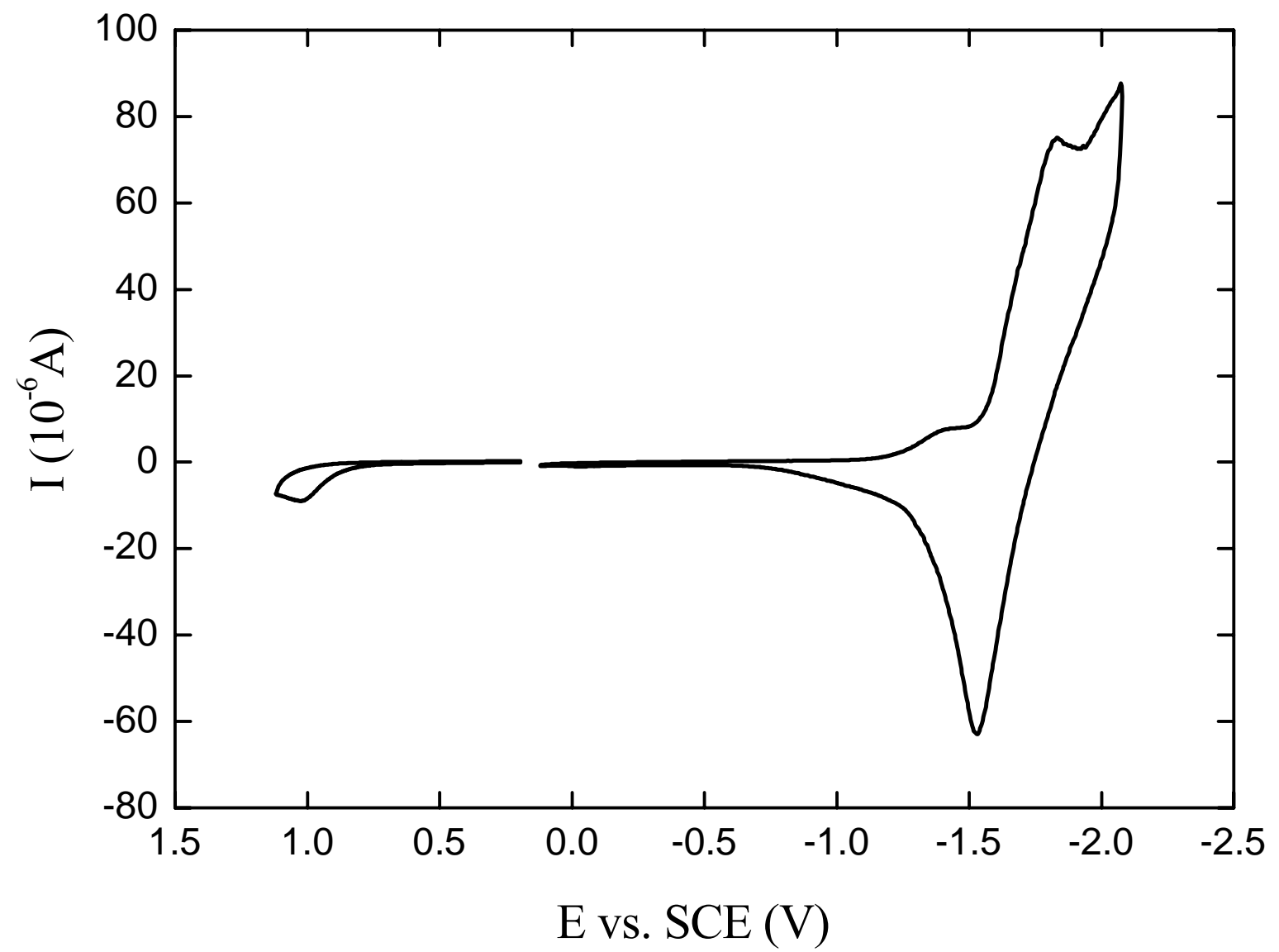

Figure S2. The cyclic voltammogram of PHTPI. 


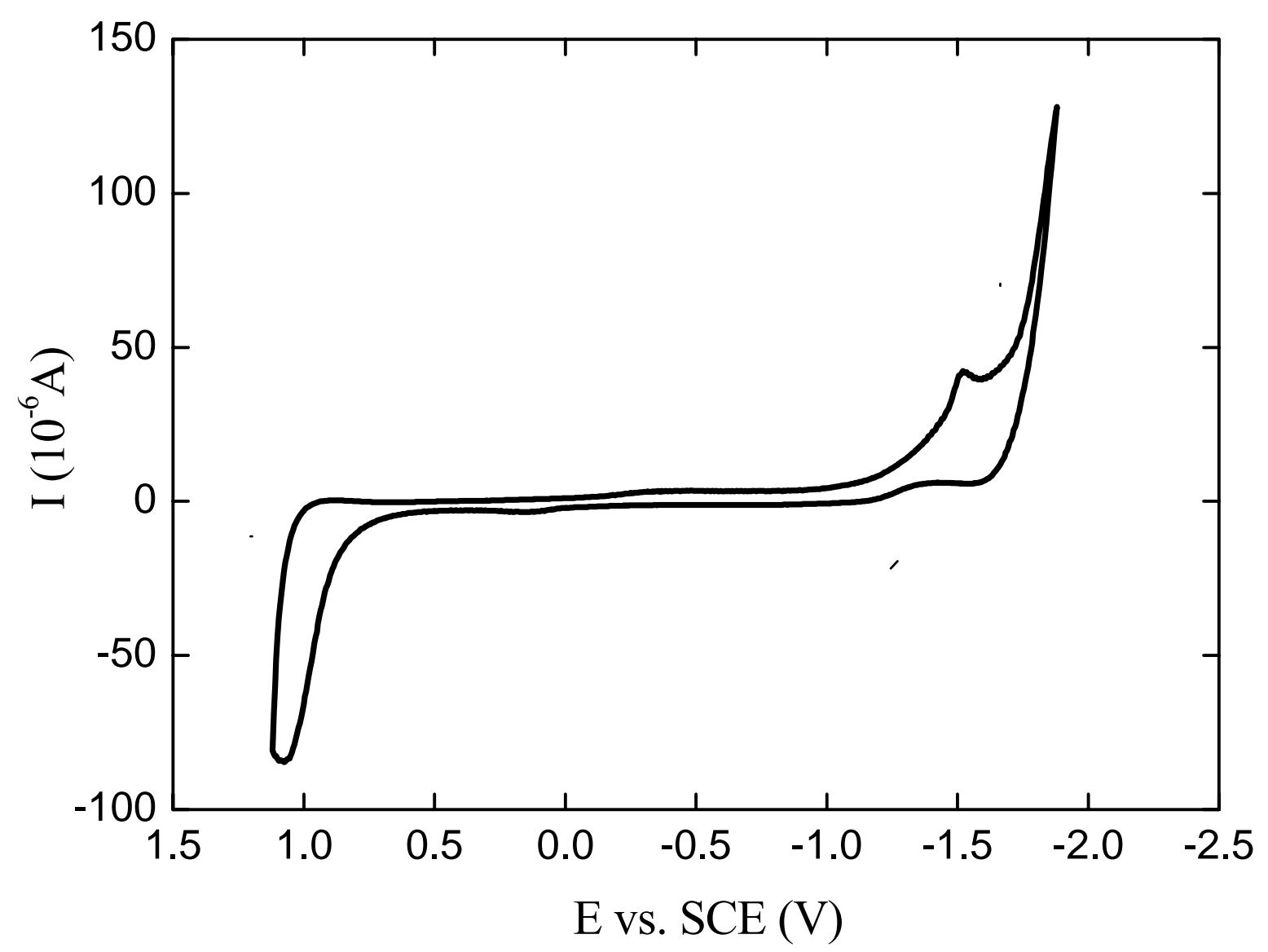

Figure S3. The cyclic voltammograms of PHTFI. 


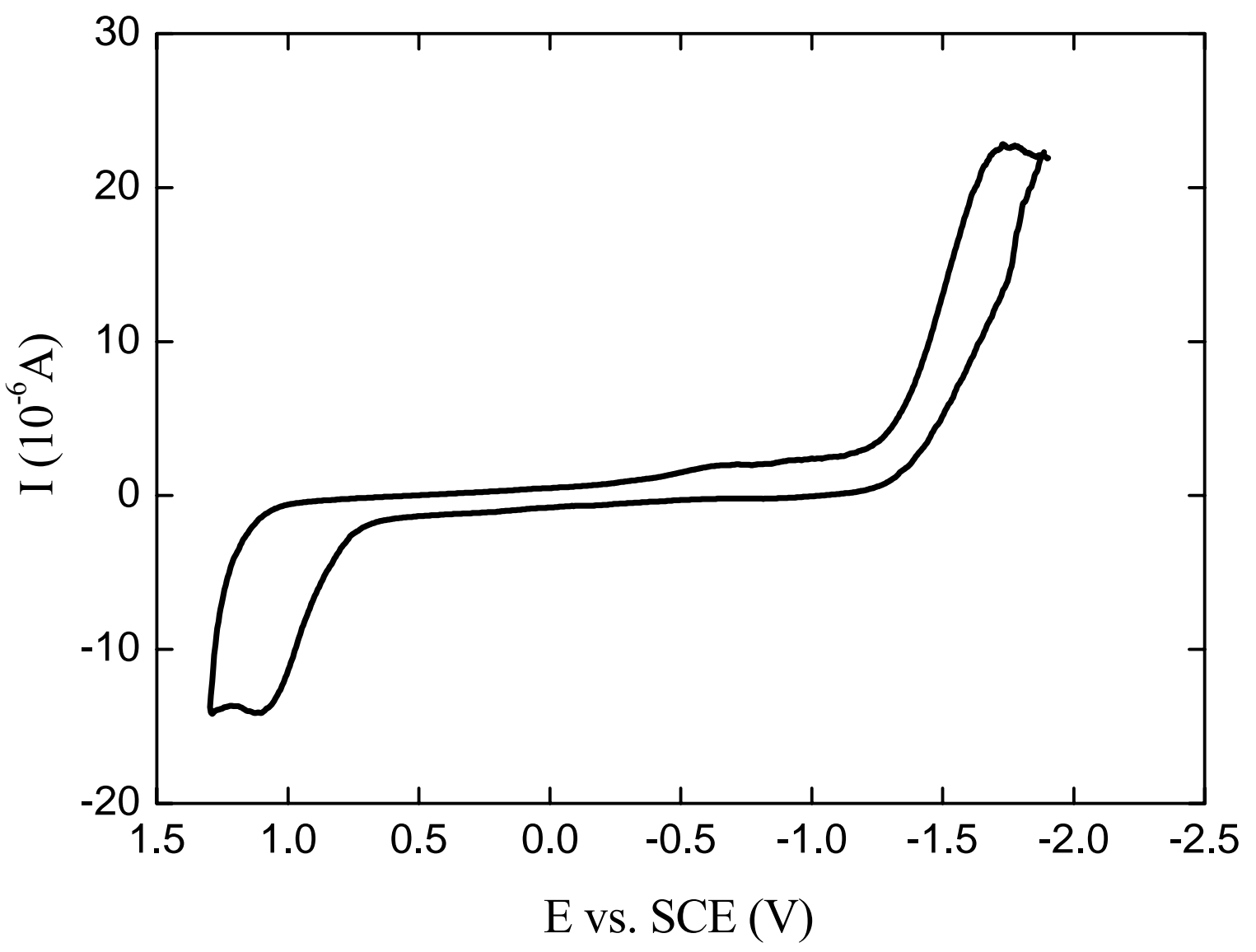

Figure S4. The cyclic voltammograms of PHTCI. 


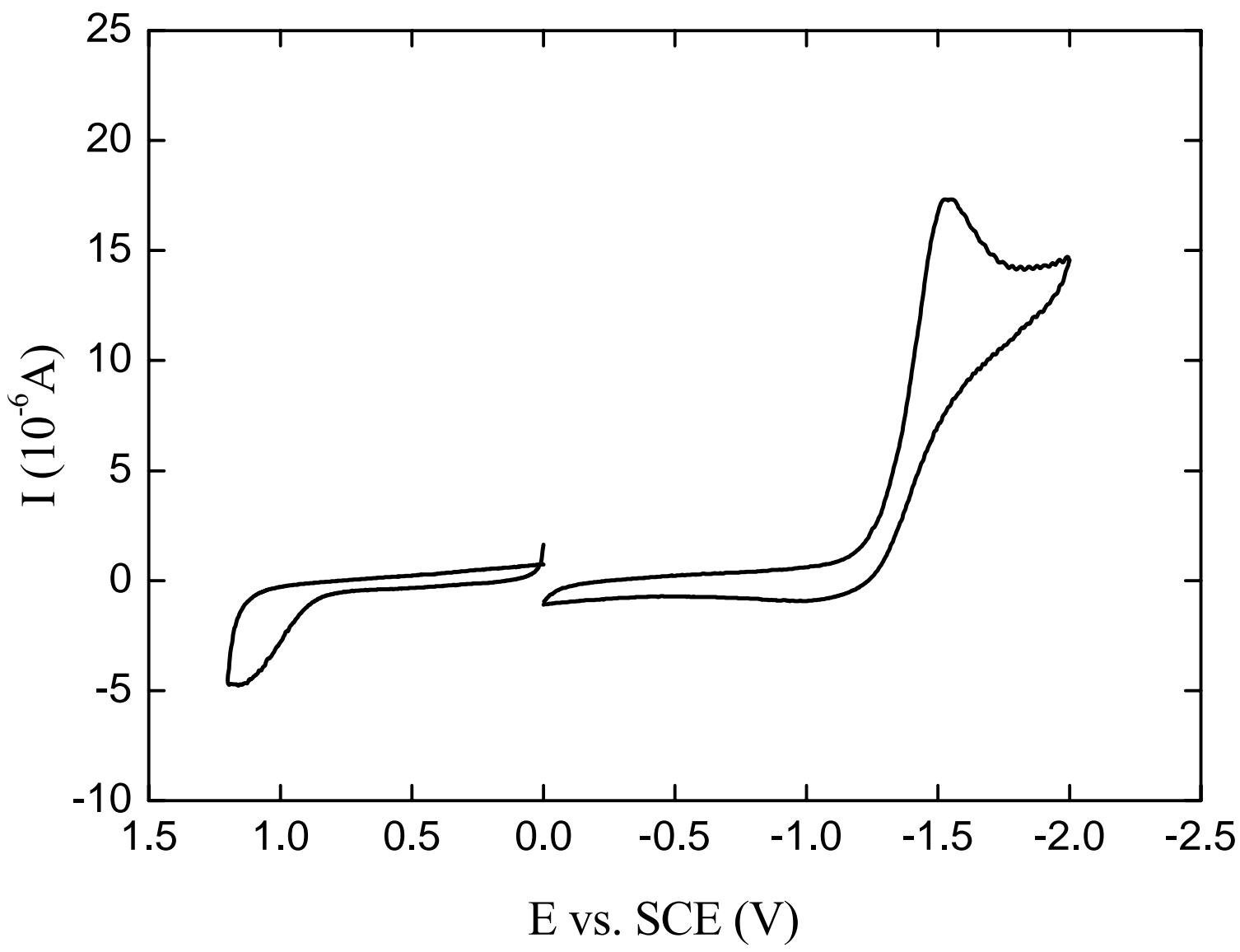

Figure S5. The cyclic voltammograms of PHTNI. 